Ann. Zootech., I976, 25 (3), 337-349.

\title{
EFFET DU FLOCONNAGE SUR LES MODALITÉS DE LA DIGESTION ET LA VALEUR NUTRITIVE DU MAÏS POUR LE PORC EN CROISSANCE
}

\author{
I. I'. BORGIDA \\ avec la collaboration technique de Moniqque DIEz, Françoise l)udot, \\ Françoise Kozlowski. S. Geeneau et G. Lecannu \\ Laboratoiro de Technologie des Aliments des Animaux, \\ Centre national de Recherches zootechniqu's, $1 . N$. R. A., \\ 78350 Jouy en Josas
}

\section{RÉSUMÉ}

En vue d'apprécier l'intérêt du floconnage du maïs pour le Porc en croissance, une comparaison de la céréale traitée au témoin correspondant est effectuée au moyen de mesures d'efficacité énergétique et azotée par la méthode des bilans ou d'analyses des contenus digestifs d'animaux abattus après un repas test.

A partir des bilans établis sur des porcs de $30-50 \mathrm{~kg}$, recevant un régime à 12 ou $15 \mathrm{p}$. 100 de protéines équilibrées, on montre que le floconnage conduit à :

I. une amélioration significative de l'utilisation digestive de tous les constituants, notamment de l'azoté du régime. et,

2. une élévation de la rétention azotée $\left(+0,7 \mathrm{~g} \mathrm{~N} / \mathrm{kg}^{\mathrm{po}} \mathrm{P}^{0}\right)$ au seul niveau hypoazoté,

3. un accroissement ( $\div+$ p. Ioo) de la teneur en énergie métabolisable de la matière sèche du maïs.

Par abattage de 20 porcs, une et trois heures après un repas test à bas taux azoté (traceurs $\mathrm{PEG}$ et $\mathrm{Cr}_{2} \mathrm{O}_{3}$ ) et mesures des $\mathrm{pH}$, de la composition, et de l'activité $\alpha$-amylasique des contenus de l'estomac, de l'intestin grêle et du cécum, il a pu être montré avec le maîs floconné, que :

I. la vidange stomacale de l'eau est fortement ralentic et s'accompagne d'une persistance d'activité amylasique ainsi que d'une chute plus tardive mais plus accentuée du $\mathrm{pH}$ dans cet organe

2. la dégradation stomacale de l'amidon et des protéines est accruc. L'amylolyse intestinale provoque la formation de glucides solubles de différentes tailles à des concentrations io à 30 fois supérieures à celles clu témoin.

3. l'amidon du mais floconné ne parvient pas au crocum. 


\section{IN'TRODUC'TION}

Les céréales destinées à l'alimentation des animaux stubissent des traitements préalables, broyage, concassage, aplatissage, qui en améliorent l'efficacité nutri.tionnelle; mais celle-ci pourrait encore être accrue par une action de la chaleur humide comme dans le floconnage.

Ce procédé, qui comporte un aplatissage de la graine à la suite d'une cuisson, est en faveur dans l'alimentation de l'Homme. Chez le Porc, très peu de recherches ont été effectuées sur les produits de cette technologie et les résultats en sont quelque peu contradictoires. WOODMANN (I925), WOODMANN et EvaNs (I932) font état d'une très importante amélioration de la digestibilité de la matière organique $(+8$ points) et de l'azote (de + I5 à + I 7 points) du maìs après floconnage, mais cet effet n'est retrouvé ni par SHEEHY et SENIOR (I939), ni par LAWRENCE (I968).

Les essais d'efficacité alimentaire n'ont pas non plus clairement montré l'intérêt du floconnage du maiss, puisque BurnetTt et NEII, (1964) ne mettent en évidence que de faibles différences ( $+3 \mathrm{p}$. Ioo), confirmées par les données de $\mathrm{L}, 4 \mathrm{~W}$ RENCE (I972 a); pour leur part, HINES et ALLEE (I972) notent une amélioration de Io p. Ioo de l'indice de consommation.

Aucun auteur ne trouve d'effet sur la rétention azotée de régimes à bas (WoodMANN, EVANS, I932) ou haut (LAWRENCE, I972 b) niveau azoté. LAWRENCE (I972 a), dans une revue, fait état des risques accrus d'action ulcérogène sur les estomacs de porcs sous l'effet de traitements hydrothermiques et note en contrepartie la possi. bilité d'avantages sur le plan de la qualité microbiologique. Cet auteur remarque également (LAWRENCE, I g68) que le floconnage du maïs augmente le pourcentage de morceaux maigres de la carcasse; il attribue cet effet à une diminution de la teneur en matière grasse des grains par dégermage partiel au cours du floconnage.

Pour préciser la valeur nutritionnelle controversée du maïs floconné et évaluer le rôle de rétention d'eau et de modification de la texture que joue cette céréale lorsqu'elle est distribuée en pâtée au Porc en croissance, nous avons essayé de séparer l'effet du traitement du maïs, d'une part sur le bilan azoté, d'autre part sur l'utilisation énergétique des régimes, mesurés selon une méthodologie classique à deux niveaux protéiques différents, puis de suivre, par des abattages successifs après un repas test, le passage et la dégradation des aliments dans le tube digestif.

\section{MATÉRIEL ETT MÉTHODES}

\section{1. -- Technologie et composition des céréales}

Le traitement de floconnage du maïs, ainsi que son effet sur la composition et les caractéristiques des fractions glucidiques de la céréale sont décrits dans un autre mémoire (DELORTLaval et Mercier, I976). Le test de digestibilité des matières azotées in vitro par la pepsine chlorhydrique est effectué sclon la méthode officielle (Anonyme, I972). Certaines fractions azotées de l'aliment et des contenus digestifs ont été séparées et dosées selon une méthode précédemment décrite (Borgida, Delort-Laval, Virobex, i 97.5). 


\section{B. - Bilan sur le Pore en croissance}

\section{Animaux.}

L'essai porte sur huit porcs mâles castrés de race Large' White, issus de trois portées contemporaines et d'un poids compris entre 25 et $29 \mathrm{k} g$ au début des essais.

\section{Alincutation.}

La base des régimes est constituée (pour sij p. 100) par la céréale broyée ténoin (I) ou floconnée (F). Elle est complétée par 6 ou $\mathrm{I} \geq$ p. Ioo de farine d'anchois du Pérou dépourvue de glucides, selon le taux de matières azotées totales recherché (H: i5,5 p. I00; 3 : i 2 p. roo). Cet apport azoté est complété, au taux protéique le plus faible, par une addition de o, I 2 p. Ioo de lysine sous lorme chlorhydrate, en vue d'établir au même niveau le rapport entre lysine et acides aminés soufrés dans tous les régimes.

Les aliments contiennent également 3 p. 100 de cellulose purifiée qui a scrvi de support de traceur et + p. Ioo de complément minéral et vitaminique qui fournit :

en glkg d'aliment

$$
\begin{aligned}
\mathrm{I}^{3} & : 4,4 \\
\mathrm{Na} & : 2,0 \\
\mathrm{Ca} & : 8,6 \\
\mathrm{Mg} & : 0,36 \\
\mathrm{Cu} & : 0,006 \\
\mathrm{Zn} & : 0,026 \\
\mathrm{Mn} & : 0,03 \\
\text { Ie } & : 0,036
\end{aligned}
$$

en $m g / k g$ d'aliment

$$
\begin{aligned}
\text { thiamine } & : 7,5 \\
\text { riboflavine } & : 15 \\
\text { acide nicotinique } & : 27,5 \\
\text { acide folique } & : 37,5 \\
\text { acide } p \text {-aminobenzoïque } & : 50 \\
\text { inositol } & : 2,5 \\
\text { biotine } & : 0,3 \\
\text { pantothénate Ca } & : 85 \\
\text { choline } & : 626
\end{aligned}
$$

$$
\begin{gathered}
\text { en LI/hg d aliment } \\
\text { vit. A }: 5,000 \\
\text { D } 3: 4500 \\
\text { E: }: 250
\end{gathered}
$$

Les résultats d'analyse des régimes sont consignés au tableau I.

\section{TABLEAU I}

Caractéristiques moyennes des régimes* (en p. roo du produit)

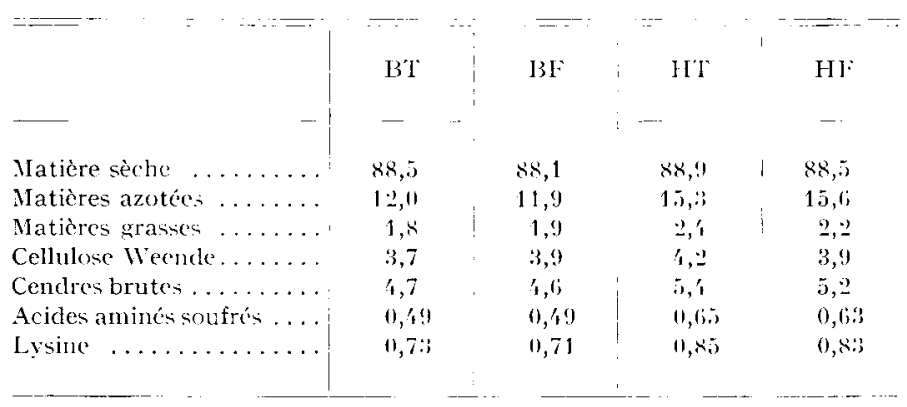

* Niveau azoté bas (B) ou haut (II). Céréale témoin (T) ou floconnéc (F).

Ia ration journalière est clistribuéc en deux repas égaux sous forme de pâtéc à environ $28 \mathrm{p}$. Ioo de matière sèche (MS) à raison de $40 \mathrm{~g}$ MS par $\mathrm{kg}$ de poids vif. Elle est régulièrement ajustéc à ce taux en fonction du poicls des animaux, enregistré chaque semaine durant l'expérience. 


\section{Déroulenent de l'essai.}

Les animaux sont placés en cages à métabolisme individuclles (FARRIEs et OsLAGl, I96I). La mesure du bilan est effectuée sur deux périodes successives de 8 jours, précédées chacune de Io jours d'adaptation.

Durant la première période, chacun des quatre groupes de deux animaux reçoit une céréale (' 1 ou $\mathrm{F}$ ) et un taux azoté (B ou H). Dans lá deuxième partie de l'essai, 1'un des animaux de chaque groupe change de céréale, l'autre de niveau azoté.

Les conditions de pesée, de collecte des excreta et de leur analyse sont celles décrites précédemment (Borgida, Delort-Laval, Viroben, 1975). La teneur en énergie des échantillons du bilan est déterminée par combustion en bombe calorimétrique adiabatique. La créatinine urinaire est dosée par la méthode colorimétrique de Jaffé ('TAussky, I956). I'ar analyse de variance (Cochran et Cox, I 966) sont séparés les effets du floconnage, du niveau azoté ct leur interaction.

\section{C. - Collecte des contenus digestifs}

Les contenus digestifs sont prélevés sur vingt porcs d'un poids de $45-50 \mathrm{~kg}$ aliattus une ou trois heures après un repas test.

Celui-ci est constitué du régime BF ou BT auquel est ajouté o,9 p. roo d'oxyde de chrome absorbé sur cellulose purifiée (Tisseran1), Coleou, Zelter, I962) et iog de polyéthylèneglycol 4 ooo (PEG) en solution aqueuse. Ce dernier traceur, ainsi que les fractions glucicliques extraites par des mélanges éthanol-eau à 80 et fo $0^{\circ} \mathrm{GI}$, sont dosés, par colorimétrie, selon des techniques déjà décrites (Borgida, Delort-Laval, Viroben, 1975). L'oxyde de chrome est dosé selon Strverson et Clare (I962), l'activité $\boldsymbol{x}$-amylasique selon Ceska, Birath et JRowr (1969).

\section{RÉSULTATS}

Les caractéristiques de composition des céréales et les effets biochimiques du floconnage sur l'amidon sont analysés ailleurs (DELORT-LAVAI, et MERCIER, I976) ; le traitement accroit fortement la fraction glucidique soluble et la sensibilité de l'amidon à l'attaque par une alpha-amylase bactérienne; il joue peu par contre sur la solubilité des protéines du maïs, déjà faible dans le maïs broyé (64 p. Ioo d'azote insoluble); le test de solubilisation in vitro des protéines par la pepsine ne montre que des effets peu prononcés du traitement sur le taux d'azote soluble ( $\mathrm{T}$ : 90 p. Ioo; F : 92,5 p. I00).

$$
\text { A. - Étude de bilans }
$$

\section{Bilan digeslif et métabolique de l'azote.}

Les données détaillées du bilan azoté sont fournies au tableau 2 ; l'utilisation digestive apparente des régimes (CUI)) et les critères de l'efficacité azotée sont regroupés au tableau 3.

Dans le cas du régime à $\mathrm{I} 5 \mathrm{p}$. Ioo de matières azotées totales, l'effet principal du floconnage du maïs est une élévation sensible de leur coefficient d'utilisation digestive par rapport à un régime témoin dans lequel elles sont, il est vrai, peu digestibles ( $m \pm s ; 74,2 \pm \mathbf{r}, 6)$. L'effet sur l'utilisation métabolique est négligeable et globalement non significatif.

Au bas taux azoté, on retrouve, accentué ( + I 2 points) ce même effet sur l'utilisation digestive des protéines brutes; mais celui-ci s'accompagne d'une élévation de $20 \mathrm{p}$. Ioo du bilan rapporté à la taille métabolique. Cette amélioration va de pair avec l'accroissement de la créatininurie (tabl. 3). 
TABLEAU 2

Résultats moyens journaliers du bilan par période ct par animal

\begin{tabular}{|c|c|c|c|c|c|c|c|c|}
\hline Régime & Poids & Gain & MS & Ingérée & \multicolumn{4}{|c|}{$N(g / j)$} \\
\hline période (I oll II) & $(\mathrm{kg})$ & $(\mathrm{kg} / \mathrm{j})$ & $(\mathrm{kg} / \mathrm{j})$ & $\left(\mathrm{g} / \log \mathrm{P}^{0,75}\right)$ & Ingéré & Urinaire & Fécal & Bilan \\
\hline BT I & 33,3 & 0,46 & 1,27 & 91,2 & 28,6 & 9,6 & 7,5 & 11,5 \\
\hline $2 \mathrm{I}$ & 30,8 & 0,36 & 1,20 & 91,3 & 27,0 & 10,0 & 7,6 & 9,1 \\
\hline $8 \mathrm{II} \ldots$ & 41,6 & 0,57 & 1,56 & 95,0 & 34,5 & 11,7 & 8,4 & $14,{ }^{\prime}$ \\
\hline $6 I I \ldots \ldots \ldots \ldots$ & 50,5 & 0,67 & 1,96 & 103,6 & 42,9 & $1: 7$ & 11,2 & 17,1 \\
\hline $\mathrm{BF} G \mathrm{I}$ & $39, \underline{-}$ & 0,63 & 1,19 & 94,6 & 32,3 & 11,6 & 4,1 & 16,4 \\
\hline $10 \mathrm{I}$ & $33, \underline{2}$ & 0,56 & 1,24 & 89,7 & 27,0 & 9,7 & 3,8 & 13,6 \\
\hline $1 \mathrm{II}$ & 42,4 & 0,66 & 1,57 & 97,0 & 33,6 & 11,5 & 4,6 & 17,6 \\
\hline 7 II .. & 42,5 & 0,57 & 1,58 & 95,1 & 33,7 & 13,1 & 4,7 & 15,9 \\
\hline $\mathrm{HT} \& \mathrm{I}$ & 32,5 & 0,52 & 1,22 & 89,8 & 33,1 & 11,2 & 9,0 & 13,0 \\
\hline $9 \mathrm{I}$ & 31,5 & 0,57 & 1,17 & 87,7 & 31,7 & 10,2 & 6,7 & 14,8 \\
\hline $2 \mathrm{II}$ & 40,1 & 0,69 & 1,46 & 91,6 & 39,6 & 13,9 & 9,1 & 16,6 \\
\hline$t_{\mathrm{t}} \mathrm{II} \ldots$ & $4 \geq 2,1$ & 0,74 & 1,65 & 99,8 & 44,8 & 18,5 & 8,9 & 22,4 \\
\hline $\mathrm{HF} \& \mathrm{I}$ & $31, \underline{1}$ & 0,53 & 1,16 & 88,0 & 30,0 & 11,3 & 5,2 & 13,6 \\
\hline $7 \mathrm{I} \ldots$ & 32,5 & 0,52 & 1,19 & 87,6 & 30,8 & 12,1 & 5,1 & 13,7 \\
\hline $10 \mathrm{II} \ldots$ & 44,7 & 0,78 & 1,64 & $9 ;, 8$ & 43,0 & $36, ' t$ & 6,1 & 20,6 \\
\hline $9 \mathrm{II} \ldots \ldots \ldots \ldots$ & 42,5 & 0,71 & 1,55 & $93, \mathbf{1}$ & 10,7 & 15,6 & 5,7 & 19,1 \\
\hline
\end{tabular}

TABLEAU 3

Utilisation digestive apparente des régimes $(n=8)$ et bilan azoté $(n=4)$

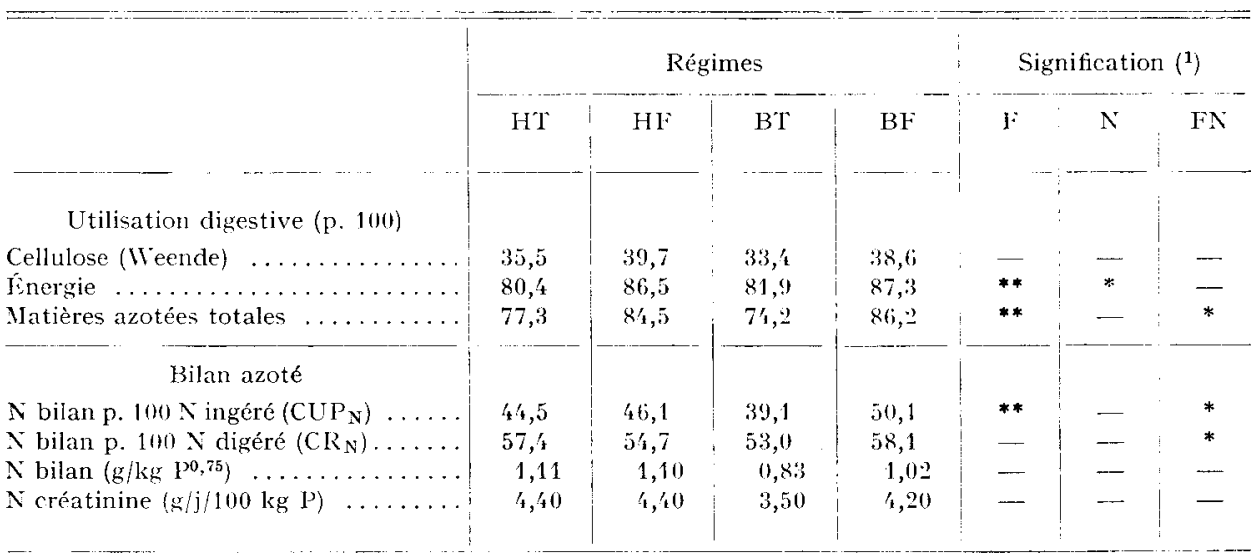

(1) Effet statistique; F du floconnage $\mathrm{N}$ du niveau azoté If $x$ interaction
* P 0,05.

** P 0,01. 


\section{C'ilisation dige'stive et métabolique de l'énergie'.}

La meilleure utilisation digestive $(\mathbf{P}<0,0 \mathrm{I})$ de l'énergie s'explique en partie par celle des matières azotées totales. Les valeurs relativement faibles des coefficients d'utilisation digestive de l'énergie des régimes sont dues à la présence de 3 p. Ioo de cellulose purifiée très peu digestible.

La fraction métabolisable de l'énergie ingérée, qui est de 79,4 et 77,9 p. Ioo respectivement avec $\mathrm{B}^{\prime} \mathrm{T}$ et $\mathrm{H}^{\prime} \mathrm{I}$, passe à 85 , I et 83,9 respectivenent avec $\mathrm{BF}$ et $\mathrm{HF}$. $I_{\gamma}$ 'écart entre les valeurs obtenues à chacun des deux taux azotés est faible ( 1,2 à $\mathrm{I}, 5$ points) et on peut donc sur ces bases estimer à $3660 \mathrm{kcal} / \mathrm{kg}$ MS la teneur en énergie métabolisable du maïs témoin et à $38 \mathrm{r} 0 \mathrm{kcal} / \mathrm{kg}$ IS celle du floconné. Après correction de l'énergie métabolisable pour la rétention azotée $(8,73 \mathrm{kcal} / \mathrm{gN}$ retenu), on calcule des valeurs fourragères de $I, 26$ et $I, 39 \mathrm{UF} / \mathrm{kg}$ MS respectivement pour les maïs témoin et floconné.

L'utilisation digestive de l'énergie n'est que faiblement (- I,5 point) mais significativement réduite par l'accroissement du niveau azoté. Il n'apparaît pas d'interaction entre ces deux facteurs.

\section{Interaction entre l'effet du nivean azoté du régime et celui du floconnage du maïs.}

Le supplément azoté, s’il améliore ( + 3, I points) la digestibilité apparente de l'azote des régimes contenant le maïs T n'a pas d'effet avec $F$. Pour tous les critères digestifs ou métaboliques du bilan azoté, il en résulte une interaction négative, bien mise en évidence par la comparaison des rapports entre matières azotées digestibles (MAD) et énergie nette, calculées d'après les données de bilan aux deux niveaux azotés ; de $9 \mathrm{I}$, I et $93,5 \mathrm{~g} \mathrm{MAI} / \mathrm{UF}$ pour BT et $\mathrm{BF}$, on passe à I I 7 et I I I g MAI/UF pour HT et HF.

\section{B. - Analyse des continus digestifs}

I. Eau, matièrc sèche, $p H$.

Le floconnage du maïs modifie le pH des contenus digestifs (de l'estomac au cæcum, gros intestin exclu) et leur teneur en eau. A l'aliment BF correspond une teneur en MS inférieure de plus de 15 points dans l'estomac et de plus de 3 points dans les deux tiers distaux de l'intestin grêle et le cæecum par rapport à celles mesurées avec le régime témoin (BT).

Le contenu total de matière sèche varie peu durant les trois heures où il a été mesuré et correspond à environ $70 \mathrm{p}$. Ioo de l'ingéré avec B'I et seulement $45 \mathrm{p}$. Ioo avec $B F$. La part de la matière sèche présente dans l'estomac reste aux environs de $60 \mathrm{p}$. Ioo du contenu digestif sec total, défini ci-dessus, quel que soit le régime.

La quantité d'eau retrouvée correspond environ, quel que soit le régime, à 75 p. Ioo de celle ingérée une heure avant et à 80 p. IOo, trois heures arant. Cependant, $35 \mathrm{p}$. Ioo de l'eau totale des contenus, dans le cas du régime $\mathrm{T}$, et près de 50 p. Ioo, dans celui de $\mathrm{F}$, sont présents dans l'estomac.

Au cours de la digestion du maïs floconné, le $\mathrm{pH}$ du milieu intestinal reste plus bas qu'avec le témoin, sauf dans l'estomac une heure après le repas. I)ans cet organe, le $\mathrm{pH}$ passe de 5,8 et 4,8 avec les régimes $\mathrm{BF}$ et $\mathrm{B}$ 'T, une heure après le repas, à respectivement 4,0 et 4,5 après 3 heures. Cette acidification plus forte chez les ani- 
maux nourris au maïs floconné se répercute sur le pH du premier tiers de l'intestin oì l'on note un $\mathrm{pH}$ aussi bas que 4,9 (contre 6 , I pour le témoin), trois heures après le repas.

\section{Marquours du transit (fig. I).}

Le taux de récupération moyen des traceurs ingérés est bon pour l'oxyde de chrome (92 p. I00), médiocre pour les polyéthylèneglycol (70 p. I00). Les pertes de ce marqueur sont dues à une fraction adsorbée ou absorbée par la muqueuse (estimée entre to et 20 p. Ioo de l'ingéré) supérieure à celle $d u C_{2} \mathrm{O}_{3}$, ainsi qu'à son passage probable mais non contrôlé, dans le gros intestin.
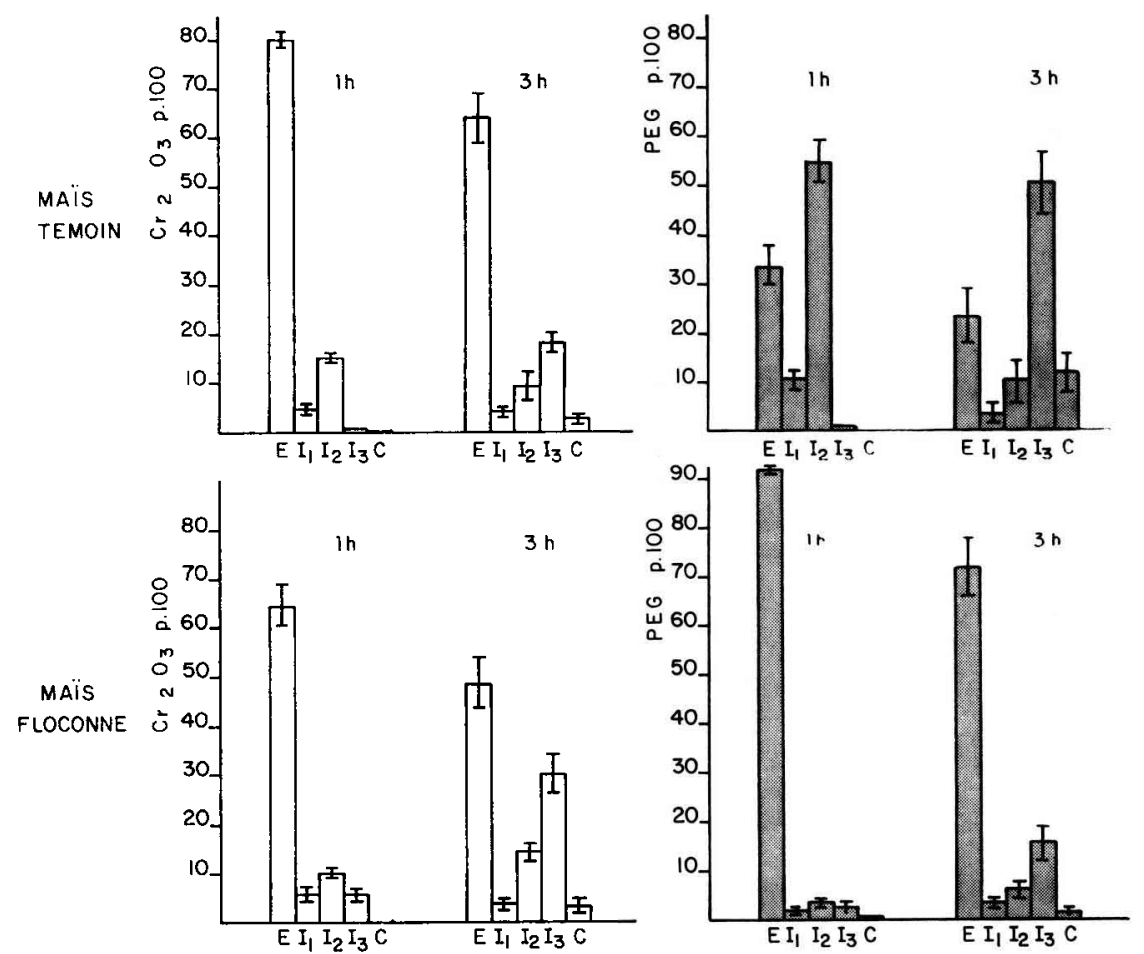

FIG. I. - Répartition des marqueners vetrouvés dans les contenus digestifs du Porc $\left(m \leq s_{m} ; n=5\right)$

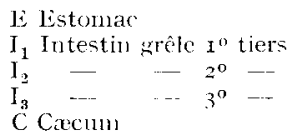

Le marquage, par l'oxyde de chrome, de la cellulose qui transite avec la matière sèche, ne montre pas de différences significatives entre les régimes, en ce qui concerne la vidange stomacale et l'accumulation progressive de ce traceur dans le second et le troisième tiers de l'intestin grêle et dans le cæcum.

Comme le montre l'examen des teneurs en PEG des contenus, le floconnage retarde fortement la sortie des liquides de 1'estomac; 73 p. IoO du PEG ingérés 
sont trouvés dans l'estomac une heure après le repas, 60 p. Ioo après trois heures, alors qu'avec le maïs normal, les taux correspondants sont de 23 et I 8 p. roo. Par la suite, le transit de l'eau dans l'intestin grêle est synchrone de celui de la cellulose et de l'oxyde de chrome.

\section{Activité $\alpha$-amylasique.}

Les activités alpha-amylasiques mesurées dans les différents compartiments digestifs sont très variables, d'un animal à l'autre et d'un régime à l'autre, et ne s'expriment donc commodément qu'en unités logarithmiques.

De l'examen de ces données (tab1. 4), il ressort que l'activité totale de l'enzyme des contenus (gros intestin exclu) reste assez constante dans les premières heures de la digestion; mais, au niveau stomacal, elle est beaucoup plus faible et diminte plus vite avec le maîs normal qu'avec le floconné. A l'inverse, dans le cæcum, elle est plus élevée avec le maïs témoin.

\section{TABLEAU 4}

Activité $\propto$ amylasique soluble présente. aux différents niveaux du tube digestif $\left(\frac{\mathrm{I}}{n} \Sigma \log \mathrm{LI} \pm s_{m} ; n=5\right)$

\begin{tabular}{|c|c|c|c|c|c|c|c|}
\hline \multirow{2}{*}{ Réginue } & \multirow{2}{*}{$\begin{array}{c}\text { Temps } \\
\text { (h) }\end{array}$} & & \multicolumn{3}{|c|}{ Tiers de l'intestin grêle } & \multirow{2}{*}{ Carcum } & \multirow{2}{*}{$\begin{array}{c}\text { Total } \\
\left(\mathrm{UI} \cdot 10^{3}\right)\end{array}$} \\
\hline & & & 1 & 2 & 3 & & \\
\hline \multirow[t]{2}{*}{ Maiss témoin } & 1 & $1,98 \pm 0,12$ & ${ }^{4,39} \pm 0,22$ & $5,05 \pm 0,11$ & $3,90 \pm 0,19$ & $2,81 \pm 19,05$ & 178 \\
\hline & 3 & $0,46 *$ & $3,87 \pm 0,18$ & $4,38 \pm 0,{ }^{\prime} 4$ & $4,17 \pm 0,17$ & $3,21 \pm 0,12$ & 157 \\
\hline \multirow{2}{*}{ Maïs floconné } & 1 & $3,55 \pm 0,21$ & $4,86 \pm 0,18$ & $4,48 \pm 0,21$ & $4,28 \pm 0,33$ & $2,28 \pm 0,21$ & 190 \\
\hline & 3 & $3,41 \pm 0,07$ & $4,35=0,31$ & $4,72 \pm 0,12$ & $4,8: \pm 0,10$ & $2,59+0,17$ & 181 \\
\hline
\end{tabular}

* Valeurs négligeables.

\section{Digestion de l'amidon.}

L'amidon représente 67,4 et $65,0 \mathrm{p}$. Ioo de la matière sèche (MS) des régimes $\mathrm{BF}$ et B'T. Les quantités retrouvées une et trois heures après le repas ne diffèrent pas significativement; elles sont nettement plus élevées dans l'ensemble des compartiments digestifs avec le mais floconné.

La digestion de l'amidon de la céréale traitée est, de beaucoup, la plus rapide; une heure après, la concentration de l'amidon dans la MS stomacale est de 45,4 p. Ioo contre 60,3 avec celui de la céréale témoin et l'amidon arrivant dans l'intestestin grêle est presque entièrement digéré ; sa concentration ne dépasse pas 3,8 p. Ioo avec le témoin, il n'en reste que des traces indosables avec la céréale floconnée. De ce fait, trois heures après le repas, il ne subsiste que I 8 p. Ioo de l'amidon du maïs 
floconné ingéré dans le tube digestif, presque uniquement dans l'estomac, alors que l'amidon du mais témoin représente encore $45 \mathrm{p}$. Ioo de l'ingéré, répartis dans le tube digestif jusqu'au cæcum qui en contient 2,7 p. Ioo (tabl. 5).

TABLEAU 5

Amidon (p. Ioo de l'ingéré) vetrouvé dans les contenus digestifs

$$
\left(m \pm s_{m} ; n=5\right)
$$

\begin{tabular}{|c|c|c|c|c|c|c|c|}
\hline \multirow{2}{*}{$\begin{array}{c}\text { Maïs } \\
\text { du régime }\end{array}$} & \multirow{2}{*}{$\begin{array}{c}\text { Temps } \\
\text { (h) }\end{array}$} & \multirow{2}{*}{ Estonac } & \multicolumn{3}{|c|}{ Tiers de l'intestin grêle } & \multirow{2}{*}{ Careum } & \multirow{2}{*}{ Total } \\
\hline & & & l & 2 & 3 & & \\
\hline \multirow{2}{*}{ Témoin } & 1 & $26,3 \pm 7,8$ & $1,1 \pm 0,5$ & $2,8 \pm 0,2$ & $1 \quad 2,2 \pm 0,7$ & $2,3 \pm 0,0$ & $33, \overline{3} \pm 8,2$ \\
\hline & 3 & $35,7 \pm 7,7$ & $2,1 \pm 0,4$ & $3,8 \pm 0,8$ & $3,1 \pm 0,6$ & $2,7 \pm 1,11$ & $\therefore 2,2+10,1$ \\
\hline \multirow{2}{*}{ Floconné } & 1 & $17,8 \pm 2,0$ & 0 & 0 & 0 & 0 & $17,8=2,0$ \\
\hline & 3 & $17, \tilde{0} \pm 2,5$ & 0 & 0 & 1) & 1) & $17,9-5: 2,6$ \\
\hline
\end{tabular}

Les glucides solubles ne constituent que $\mathrm{I}, 2$ et $\mathrm{I}, 4 \mathrm{p}$. Ioo de la matière sèche des régimes $\mathrm{BT}$ et $\mathrm{BF}$ respectivement, pour les glucides les moins polymérisés, I,5 et 5.9 p. Ioo de la matière sèche pour ceux de plus haut poids moléculaire. Les concentrations de glucides solubles dans la matière sèche des contenus sont, une heure après le repas, nettement plus élevées avec le régime à base de céréale traitée (fig. 2), surtout dans l'estomac ( $224 \mathrm{mg} / \mathrm{g}$ de contenu $\mathrm{sec}$, contre 7 pour le témoin
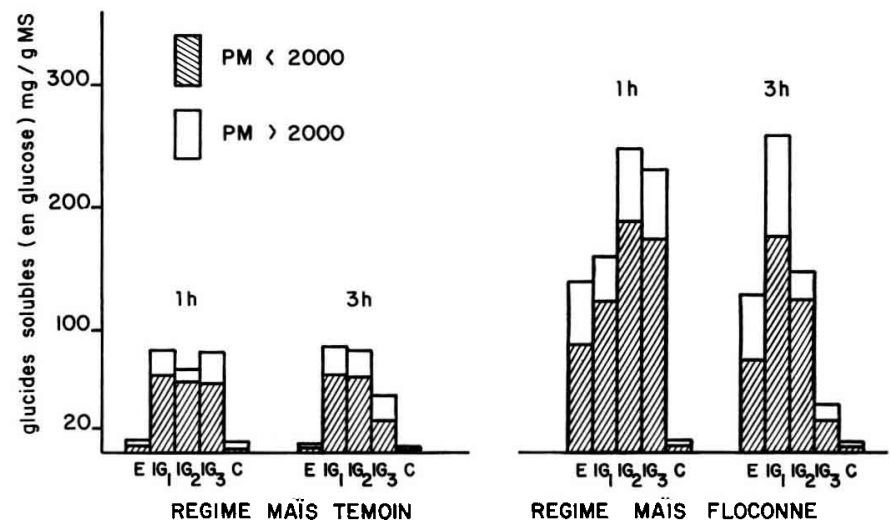

FIG. 2. - Concentration moyenne en glucides solubles dans la inatic̀re siche des contenus digestifs $(n-5)$

PMI Poirts Moléculaire

correspondant) et dans la partie distale de l'intestin grêle (234 contre 84 ). Après trois heures, les différences se maintiennent dans l'estomac et s'établissent plus nettement dans les parties proximales de l'intestin grêle pour les glucides peu polymérisés : I77 contre 7 I dans le premier tiers, I23 contre 66 dans le second, alors que, 
dans la partie distale du tube digestif, les concentrations s'abaissent et les différences entre régimes s'amenuisent. La dégradation de l'amidon de mais est fortement accrue in ino ainsi qu'on le constate dans les tests in vitro. Cette attaque amylasique rapide entraîne une accumulation, dans les contenus digestifs, de glucides solubles. Ceux-ci sont surtout composés de polyholosides de poids moléculaire inférieur à 2000 . Leur concentration correspond vraisemblablement à une limite de leur vitesse d'hydrolyse ou de l'absorption ultérieure de leurs produits de dégradation.

\section{Digestion des protéines (tabl. 6).}

Les matières azotées des régimes sont insolubles à 92 p. Ioo en solution saline. Lans l'ensemble des contenus, les teneurs moyennes en azote insoluble restent voisines de $45 \mathrm{p}$. Ioo de l'azote ingéré, alors (qu'avec le floconné, ces teneurs sont, respectivement, de 25 et $30 \mathrm{p}$. IoO, une et trois heures après le repas.

\section{TABLEAU 6}

Aute des fractions séparées dans l'aliment ingéré (temps o) et dans les contenus stomacaut do porcs ( 1 et 3 h apris le repas)

$$
\left(m \pm s_{m} ; n-5\right)
$$

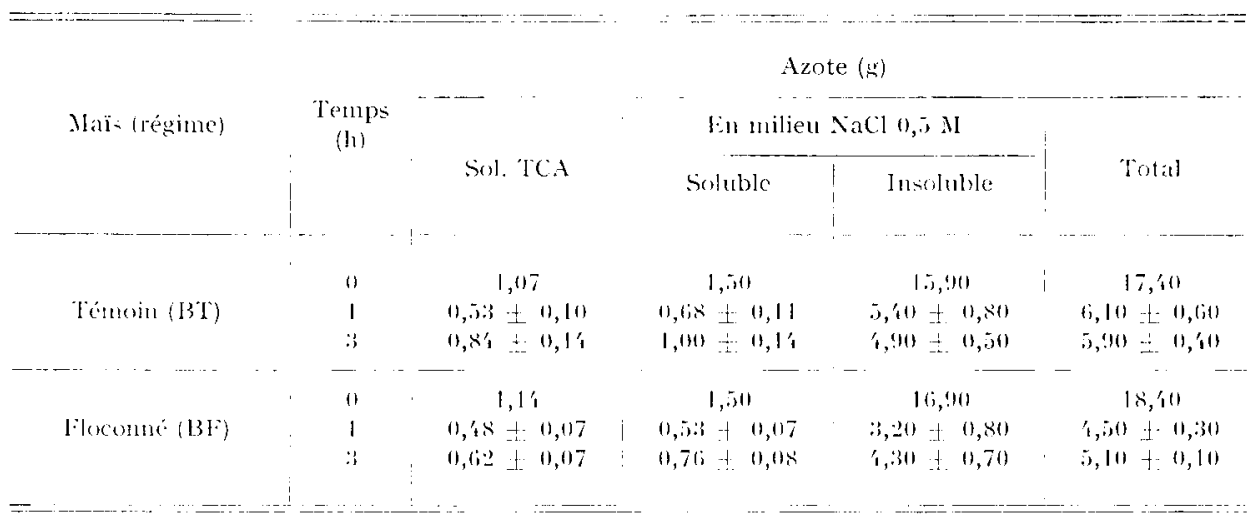

Les matières azotées totales disparaissent rapidement de l'estomac où l'on ne dose, une heure après le repas, que $36 \mathrm{p}$. Ioo de $1^{\prime} \mathrm{N}$ ingéré avec le régime témoin et $24,5 \mathrm{p}$. Ioo avec le floconné, ce qui suggère une dégradation stomacale des protéines plus forte avec ce dernier. Ainsi se confirme, malgré une variabilité élevée (coefficients de variations de 30 à 40 p. IOo) la différence des taux résiduels de protéines alimentaires dans les contenus digestifs suivant le régime.

\section{DISCUSSION E'T CONCIUSION}

Le traitement de floconnage que nous avons appliqué au maïs a eu pour effet d'améliorer très favorablement la digestibilité de ses constituants et tout particulièrement celle de l'azote du régime du Porc en croissance. Nos résultats corroborent ceux obtenus chez le Porcelet par Aumaitre. (1976) avec le même maïs floconné, 
et par WoOdman (I925), quoique cet auteur ait probablement utilisé un traitement différent du nôtre, caractérisé par une réhumidification préalable de la céréale.

L'amélioration de la digestibilité apparente des matières azotées s'accorde avec leur plus rapide disparition in vivo, constatée avec le maïs floconné, qui traduit soit une absorption plus rapide, soit une moindre sécrétion endogène. Si les données recueillies une heure après le repas dans les parties proximales du tube digestif font penser à un mécanisme de dégradation plus rapide favorisé par la rétention d'eaul et d'enzymes dans l'estomac, la forte insolubilité des protéines et la faible différence de solubilité à la pepsine in vitro plaident plutôt pour un effet de moindre excrétion azotée d'origine endogène.

De fait, en estimant à $I, 2 \mathrm{~g}$ d'azote $\mathrm{p}$. Ioo $\mathrm{g}$ de matière sèche fécale, l'azote endogène excrété (ZELTER et CHARI.ET-L,ÉRY, Ig6I) et en extrapolant les domnées obtenues aux deux niveaux azotés on peut calculer la digestibilité réelle des protéines du maïs. Elle est de $82 \mathrm{p}$. Ioo avec le témoin et de $96 \mathrm{p}$. Ioo avec le floconné. Malgré le caractère conventionnel de ce calcul il permet de montrer que la simple diminution d'excrétion fécale de matière sèche pourrait donc expliquer une bonne part des différences de digestibilité apparente des matières azotées. L'effet du floconnage sur la teneur en matière sèche des contenus digestifs et leur fermentation microbienne limitée par le fait que l'amidon de maïs floconné ne parvient pas jusqu'au niveau iléo-cæcal, vont aussi dans le sens de cette hypothèse.

Cet effet du floconnage sur la réduction de l'excrétion d'azote métabolique fécal pourrait donc être rapproché de celui de la cuisson de la fécule de pomme de terre sur la dépense azotée endogène chez le Porc, qu'observent ZELTER, CharLETLÉRY et DELORT-I,AVAL, (I966).

Malgré cette meilleure digestibilité de leurs constituants, les régimes à base de maïs floconné ne permettent pas une meilleure croissance, ni une rétention azotée plus élevée lorsque le niveau protéique est proche de l'optimum comme dans les aliments HT et HF. Les résultats de HINEs et ALLEE (I972), qui mettent en évidence une amélioration de ro $\mathrm{p}$. Ioo de l'indice de consommation, sont probablement dus, d'après les auteurs, à un plus faible gaspillage d'aliment par les porcs alimentés au maïs floconné, et ne sont donc pas en contradiction avec nos propres résultats, que confirment ceux de Burne't'T et Neil (I964), SheEhy et Senior (I939), chez le Porc en croissance, et d'Aumaitre (I976) chez le Porcelet précocement sevré.

A un taux azoté plus limitant, nous avons pu mettre en évidence une nette amélioration de la rétention azotée sous l'effet du floconnage du mais. Celle-ci est due à la fois à la meilleure digestibilité de l'azote (+ I2 points) et à une efficacité énergétique accrue, puisque la teneur en énergie métabolisable du maîs passe de 3660 à $38 \mathrm{Io} \mathrm{kcal} / \mathrm{kg}$ de matière sèche après floconnage. Comme le rapport entre matière azotée digestible et énergie métabolisable est pratiquement le même pour les deux régimes hypoazotés, on doit admettre une meilleure utilisation de l'énergie pour la rétention azotée qui est, elle, accrue de $23 \mathrm{p}$. Ioo sous l'effet du floconnage. Pour l'expliquer, il est possible de se référer au fait que l'accumulation de glucides solubles dans les contenus digestifs entraîne un accroissement de la pression osmotique et done de la teneur en eau des contenus. Cet effet, d'origine physicochimique, serait favorable à un transit digestif plus régulier et à l'absorption des nutriments à ce niveau.

De nos résultats, obtenus avec un maïs dont la teneur en azote soluble et en 
énergie métabolisable est bas, en raison d'un séchage trop intense après la récolte, il résulte que le floconnage ne peut être intéressant que pour valoriser des rations à bas taux de protéines. Son effet majeur semble être d'accélérer sensiblement le cours de la digestion des glucides et de l'azote et de limiter la dépense azotée endogène.

Pour reproduire cet effet, l'incorporation de mais floconné au régime du Porc en croissance pourrait être envisagée à des taux beaucoup plus faibles que ceux du présent essai. Des grains de qualité inférieure, en raison des conditions extrêmes de leur séchage ou de leur contamination microbiologique, pourraient être traités à cette fin. Il serait toutefois nécessaire de préciser, par des essais complémentaires, d'une part les conditions de traitement les plus favorables à la gélification de l'amidon et à l'élévation de la teneur en glucides solubles du grain, d'autre part l'effet de son taux d'introduction dans les aliments sur leur transit et l'action de la microflore digestive.

Reçu pour publication en février 1976 .

\title{
REMERCIEMENTS
}

A Ml. Vizoben, pour le fractionnement azoté des contenus digestifs et le dosage des acides aminés des rations.

\author{
SUMMARY \\ EFFECT OF FIAAKING ON THE DIGESTION PATTERN \\ AND NUTRITIVE VALUE OF MAIZE IN GROWING PIGS
}

The value of flaked maize for the growing pig was determined in nitrogen and energy balance trials and gut content analyses on animals slaughtered one or three hours after a test meal.

Balance data in $30-50 \mathrm{~kg}$ live weight pigs fed 12 or 15 p. roo protein diet, balanced in lysine and sulfur aminoacids, indicated (I) a highly significant improvement of digestibility, specially that of nitrogen; (2) a better nitrogen retention $\left(+0.7 \mathrm{~N} / \mathrm{kg} \mathrm{W}^{0,75}\right)$ with the low dietary protein level and (3) a slight increase of the metabolizable energy in the dry matter of maize $(+4 \mathrm{p}$. 100$)$.

Stomach, small intestine and caecum contents of 20 pigs slaughtered after a low protein test meal were removed and analysed for $\mathrm{pH}$, carbohydrate and nitrogen composition, markers ((PEG + ooo, $\mathrm{Cr}_{2} \mathrm{O}_{3}$ on cellulose) concentration and $\alpha$-amylase activity.

Flaking changed the meal digestion pattern during the first three hours :

(1) stomachal water emptying slowed down, amylase kept steady and a later but stronger $\mathrm{pH}$ decrease was observed in this organ ;

(2) protein and starch degradation were rapid in the stomach and amylosis extremely intense in the small intestine, where the concentration of the soluble carbohydrates of various size was ro to 30 fold higher than with the control diet;

(3) no starch appeared in the caecum.

\section{RÉFÉRENCES BIBLIOGRAPHIQUES}

Axonyme, 1972. Dosage des protéines brutes solubilisées par la pepsine et l'acide chlorhydrique. $J$. off. Commun. Europ., L $123, \mathrm{II}-\mathrm{I} 2$.

Aumaitre A., I076. Évaluation de divers traitements technologiques des céréales. IV. - Influence du floconnage et de l'expansion de l'orge et du mais sur la digestibilité des éléments de la ration et les performances du I'orcelet sevié à 2 I jours. Ann. Zootech., 25, 4I-5r. 
Jokgida L. P., Delort-I.AYal J., Viroben G., I975. Effet du mode de conservation d'un maïs immature sur l'utilisation de ses glucides et de son azote chez le Porc en croissance, finition. Ann. Zootech., 24, $433-44^{6}$

Buknet (i. S., Neil 1:. L., I964. The influence of processing and of certain crude enzyme preparations on the utilisation of cereals by pig. Anim. Prod., 6, 237-244.

Criska M., Birath K., Brow' B., 1969. A new and rapid method for the clinical determination of $\alpha$-amylase activity in human serum and urine. Optimal conditions, Clin. Chem. Acta, 26, 437-444.

Cochrax II. G., Cox G. M., ig66. Experimental designs. Second edition, J. Wiley Ed., New York, 6II p.

Delort-Laval J., Mercirk C., I976. Évaluation de divers traitements technologiques des céréales. [. - Choix des traitements et étude de leur infuence sur la fraction glucidique du blé, de l'orge et dı mais. Ann. Zootech., 25, 3-12.

FARRIES F. E., OsLAGE H. J., I96r. Zur Technik langfristiger Stoffwechselversuche an wachsenden Schweinen. ZZ. Tierphysiol. Tierernähr. Futtermittelk., 16, I I-29.

Hines R. H., AlxeE G. L., r972. Flaked corn, wheat and sorghum grain for growing pigs. Kansas State Lniversity Annual Report; Swine Industry Days, 8-1o.

LAwrence T. L. J., I968. High level cereal diets for the growing finishing Pig. III. - A comparison with a control diet containing high levels of maize, flaked maize, sorghum, wheat and barley. $J$. Agric. Sci., $70,287 \cdot 297$.

LAWRENCE T. L. J., I972 a. A review of some effects on health and performance of variations on the physical form of the diet of the growing Pig. III. - Heat treatments and cubing. Vet. Rec,, 91, IO8-I I I.

L.AWRENCE T. J. J., I972 b. Developments in cereal processing; growth Pigs. In Cereal Processing and. Digestion, ISS Feed Grain Council, Washington DC, 77-I05.

Shentr E. J., Senior B. J., r939. The comparative feeding value for pig of cereals prepared in the flaked and ground form. J. Depl. A gric. Eire, 36, 230-233.

Stevensox A. E., Clale N. T., I962. Neasurement of fecd intake by grazing cattle and sheep. IX. - Determination of chromic oxide in faeces using an autoanalyser, N. Z. J. Agric. Res, , 6, I2I-I 26.

TArssky. Fertha H., I956. A procedure increasing the specifity of the Jaffé reaction for the determination of creatine and creatinine in urine and plasma. Clin. Chem. Acta, 1, 2 Io-223.

Tisseraid J. L., Coleot J., Zelter S. Z., rg62. Emploi du sesquioxyde de chrome comme indicateur dans les études de bilan digestif chez le Ruminant. C. R. Acad. Sci. Paris, 254, 2233-2235.

Woodmanx J: H., I925. Digestion trial with swine. I. - Comparative determination of the digestibility of dry fed maize, soaked maize, cooked maize and flaked maize. J. Agr. Sci, 15, I-I 8 .

Woodmany E. H., Eivans R. E., I932. Cité par Lawrence T. L. J., I972 $b$.

Zelter S. Z., Charlet-Lerr Geneviève, Delort-Laval J., tg66. Influence de la fécule crue et cuite de pomme de terre sur les dépenses azotées métaboliques et endogènes et sur la valeur nutritionnelle d'un mélange équilibré (soja - hareng) chez le Porc en croissance. C. R. Acad. A gric. Fr., 567-573.

7elter S. Z., Charlet-Lfry (reneviève, ig6r. Ffficacité de quelques protides alimentaires chez le porc. I. - Excrétion d'azote suétabolique fécal et endogène urinaire. Ann. Biol. anim. Bioch. Biophys., 1, 29-46. 